\section{MAD2B is an inhibitor of the anaphase-promoting complex}

\author{
James Chen and Guowei Fang ${ }^{1}$ \\ Department of Biological Sciences, Stanford University,
} Stanford, California 94305-5020, USA

Anaphase-promoting complex (APC), a ubiquitin ligase, controls both sister chromatid separation and mitotic exit. The APC is activated in mitosis and $\mathrm{G}_{1}$ by CDC20 and $\mathrm{CDH1}$, and inhibited by the checkpoint protein MAD2, a specific inhibitor of CDC20. We show here that a MAD2 homolog MAD2B also inhibits APC. In contrast to MAD2, MAD2B inhibits both CDH1-APC and CDC20-APC. This inhibition is targeted to CDH1 and CDC20, but not directly to APC. Unlike MAD2, whose interaction with MAD1 is required for mitotic checkpoint control, MAD2B does not interact with MAD1, suggesting that MAD2B may relay a different cellular signal to APC.

Received March 28, 2001; revised version accepted May 22, 2001.

Proteolysis is a general mechanism that regulates critical cellular events in the cell cycle, in signal transduction, in transcription, and in development. In mitosis, both separation of sister chromatids and exit from mitosis are regulated by a proteolysis pathway. At the metaphase to anaphase transition, degradation of the anaphase inhibitor securin triggers sister chromatid separation (Amon 1999|. Later, complete degradation of mitotic cyclins inactivates Cdc2 kinase activity, leading to cytokinesis (King et al. 1995). Both securin and cyclins are degraded through a ubiquitin-dependent pathway and the key enzyme in this pathway is a ubiquitin ligase, the anaphasepromoting complex (APC)/cyclosome, named for its function in control of anaphase initiation (King et al. 1995; Sudakin et al. 1995).

The APC controls critical transitions in mitosis and tight regulation of its activity is essential for ordered progression of the cell cycle. In mitotic cell cycle, APC becomes active at the onset of anaphase, and its activity persists through most of $\mathrm{G}_{1}$ (Amon et al. 1994; Fang et al. 1998a). The activity of APC is controlled by a network of regulatory factors that include at least three proteins, MAD2, CDC20, and CDH1/HCT1 (Schwab et al. 1997; Visintin et al. 1997; Fang et al. 1998a,b; Hwang et al. 1998; Kim et al. 1998). The checkpoint protein MAD2 is an inhibitor of APC, whereas CDC20 and CDH1 are activators. Both CDC20 and CDH1 can bind directly to APC and activate its ubiquitination activity (Fang et al. 1998a).

[Key Words: Anaphase-promoting complex; CDC20; CDH1; MAD2; MAD2B; spindle assembly checkpoint]

${ }^{1}$ Corresponding author.

E-MAIL gwfang@stanford.edu; FAX (650) 725-5807.

Article and publication are at http://www.genesdev.org/cgi/doi/10.1101/ gad.898701.
Binding of CDC20 and CDH1 controls the temporal order of activation of APC in the cell cycle (Fang et al. 1999). As cells enter mitosis, phosphorylation of APC by the mitotic Cdc2 kinase enhances its affinity toward newly synthesized CDC20 and formation of the active CDC20-APC complex at the onset of anaphase triggers the degradation of securin and separation of sister chromatids. At early mitosis, the Cdc2 kinase phosphorylates $\mathrm{CDH} 1$ and prevents $\mathrm{CDH} 1$ from binding to and activating APC. Later in anaphase CDC14 dephosphorylates CDH1 (Visintin et al. 1998). The APC dissociates from CDC20 and binds to dephosphorylated CDH1. Unbound CDC20 is degraded by CDH1-APC, leading to a complete replacement of CDC20-APC by CDH1-APC. CDH1-APC also completely degrades cyclin B and inactivates the Cdc2 kinase activity, leading to exit from mitosis. At the $\mathrm{G}_{1} / \mathrm{S}$ transition, $\mathrm{CDH} 1$ dissociates from APC and the APC activity remains low in $S$ and $G_{2}$ phases of the cell cycle.

CDC20 and CDH1 also regulate the substrate specificity of the APC and may be directly involved in substrate recognition (Fang et al. 1998a). CDC20-APC requires a nine-amino-acid sequence, the destruction box (D-box), for recognition (Glotzer et al. 1991), whereas CDH1-APC recognizes the K-E-N sequence (KEN-box) (Pfleger and Kirschner 2000). Thus, the temporal regulation of the binding of CDC20 and CDH1 to APC, coupled with differential substrate recognition, may explain why different substrates are ubiquitinated at different stages of the cell cycle.

The APC is the target of the spindle assembly checkpoint, a surveillance mechanism that delays anaphase until all the chromosomes are aligned at the metaphase plate (Amon 1999). This checkpoint ensures the fidelity of sister chromatid separation by monitoring the attachment of the mitotic spindle to kinetochores and preventing premature onset of anaphase in the presence of unattached kinetochores. It has been shown that APC is under the control of the checkpoint protein MAD2, a stoichiometric inhibitor of CDC20 (Chen et al. 1996; Li and Benezra 1996; Fang et al. 1998b; Hwang et al. 1998; Kim et al. 1998). Upon activation of the spindle assembly checkpoint, MAD2 directly associates with CDC20-APC and inhibits activation of APC by CDC20 before anaphase (Fang et al. 1998b; Hwang et al. 1998; Kim et al. 1998). The MAD2 protein interacts with another checkpoint protein MAD1 and the mitotic checkpoint control requires the formation of the MAD1-MAD2 complex (Jin et al. 1998; Chen et al. 1999).

A homolog of the MAD2 protein, MAD2B, has been identified recently based on its sequence similarity to MAD2 (Cahill et al. 1999). Human MAD2B shares 25\% identity and $48 \%$ similarity to human MAD2 at the amino acid level. We show here that MAD2B is also an inhibitor of APC; it prevents activation of APC by CDH1, and to a lesser extent, by CDC20. Furthermore, this inhibition is not specific to a particular substrate, as MAD2B inhibits ubiquitination of all four substrates tested. Unlike MAD2, MAD2B does not interact with the checkpoint protein MAD1, suggesting that MAD2B may transduce a cellular signal other than the mitotic checkpoint control to the APC pathway. 


\section{Results}

\section{MAD2B inhibits degradation of cyclin $B$} in Xenopus extracts

To investigate the effect of MAD2B on the APC pathway, we expressed and purified the recombinant human MAD2B protein from Escherichia coli (Fig. 1A). Because human and Xenopus MAD2B share $95 \%$ identity at the amino acid level (Pfleger et al. 2001.; J. Chen and G. Fang, unpubl.), we analyzed the biochemical activity of purified MAD2B in Xenopus extracts. MAD2B was incubated with extracts made from Xenopus eggs and the stability of the exogenously added, radioactively labeled cyclin B protein was then analyzed. Cyclin B is stable in interphase extracts and the APC is not active (Fig. $1 \mathrm{~B}, \mathrm{C}, \mathrm{I})$. Addition of recombinant $\mathrm{CDH} 1$ protein to interphase extracts activates the APC-dependent proteolysis pathway and CDH1-treated interphase extracts degrade cyclin B with a half-life of 5-10 min, a rate comparable to that in mitotic extracts (Fig. 1B, II and VI). Degradation of cyclin B is due to direct activation of APC, as APC immunopurified from such extracts quantitatively converts cyclin B into ubiquitin conjugates in an in vitro ubiquitination assay (Fig. 1C, II). However, incubating

A

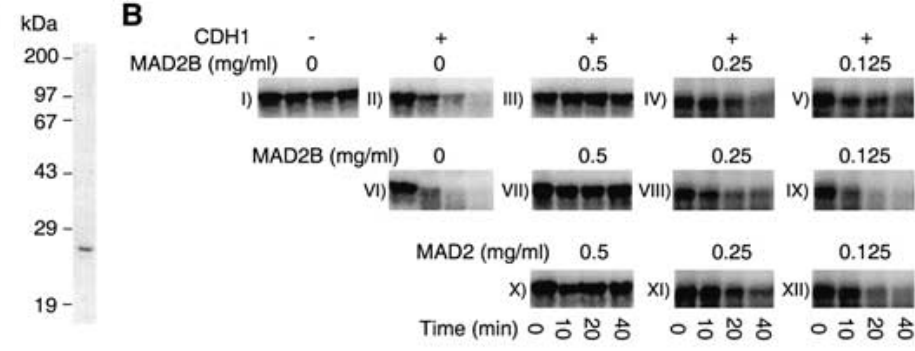

C
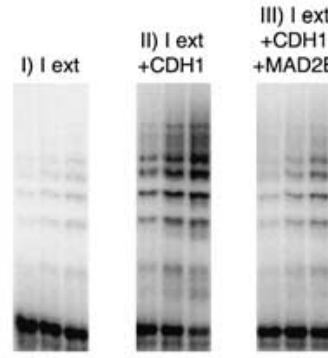

Time (min) $\vec{\circ} \tilde{\circ}$ o

$\overrightarrow{0}$ 요 0

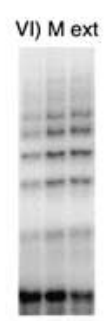

$\overrightarrow{0}$ 용

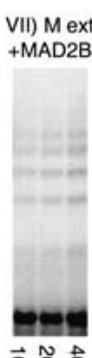

$\overrightarrow{0}$ 용

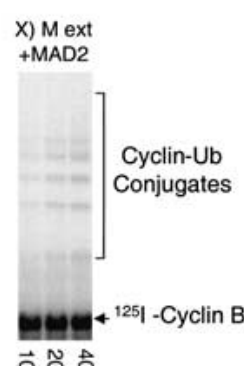

훙 fo

Figure 1. MAD2B inhibits APC activity in Xenopus extracts. (A) Purified recombinant MAD2B was analyzed by $12 \%$ SDS-PAGE. (B) Degradation of cyclin B in Xenopus extracts. $\mathrm{CDH} 1$ was incubated with Xenopus interphase extracts in the presence of various concentrations of MAD2B (II-V). Similarly, MAD2B (VII-IX) and MAD2 (X-XII) were incubated with Xenopus mitotic extracts. The stability of radioactive $\mathrm{N}$-terminal fragment (amino acids 1-102) of cyclin B was assayed in these extracts. As controls, interphase (I) and mitotic (VI) extracts were analyzed in parallel. The MAD2B and MAD2 concentrations indicated are final concentration in extracts. The recombinant MAD2 protein exists in two different forms (oligomer and monomer) and only the oligomer is an active inhibitor of APC (Fang et al. 1998b). Thus, MAD2 oligomer was used in experiments described in this paper. $(C)$ APC activity assayed in reconstituted ubiquitination reactions. APC from extracts prepared in $B$ was immunopurified by anti-CDC27 antibody/protein $\mathrm{A}$ beads and its ability to ubiquitinate $\mathrm{N}$-terminal fragment of cyclin B analyzed. (I ext), interphase extracts; (M ext), mitotic extracts.

MAD2B with CDH1 in interphase extracts at $0.5 \mathrm{mg} / \mathrm{mL}$ completely blocks degradation of cyclin $\mathrm{B}$ and activation of APC (Fig. 1B,C, III). In contrast, incubating bovine (BSA) with CDH1 in interphase extracts way.

We also analyzed the effect of MAD2B on mitotic degradation of cyclin B. Mitotic APC is fully active and mitotic extracts degrade cyclin B with a half-life of 5-10 min (Fig. 1B,C, VI). The checkpoint protein MAD2 is an ssembly checkpoint and incubation of MAD2 with miic extracts at $0.5 \mathrm{mg} / \mathrm{mL}$ inhibits the APC activity and tabilizes cyclin B (Fig. 1B,C, panels X) (Fang et al. 1998b). Interestingly, incubation of MAD2B with miinhibits APC pathway and cyclin B becomes stable in AD2B-treated mitotic extracts (Fig. 1B, VII). APC immunopurified from MAD2B-treated extracts only has an activity similar to that from interphase APC and from rol, incubating BSA with mitotic extracts has no effect on the kinetics of degradation of cyclin B (data not hown). Because APC is activated by CDC20 in Xenopus mitotic extracts (Lorca et al. 1998), these data indicate that MAD2B is also an inhibitor of the CDC20-APC pathway.

Although MAD2B can inhibit both CDC20 and $\mathrm{CDH} 1$ at a high concentration $(0.5 \mathrm{mg} / \mathrm{mL})$, MAD2B is more effective in inhibiting CDH1 at lower concentrations (Fig. 1B, III-V, VII-IX). At $0.25 \mathrm{mg} / \mathrm{mL}$ MAD2B, $75 \%$ of cyclin B was degraded within $10 \mathrm{~min}$ in MAD2B-treated mitotic extracts, whereas $<20 \%$ of cyclin was degraded within $10 \mathrm{~min}$ in MAD2B-treated CDH1 extracts (Fig 1B, cf. IV and VIII; data not shown). Similarly, at lower concentrations, MAD2B is slightly less potent than MAD2 in inhibiting mitotic degradation of cyclin B (Fig. 1B, cf. VIII and $\mathrm{XI})$.

\section{MAD2B directly inhibits activation of APC by CDC2O and CDH1}

We then examined whether MAD2B directly inhibits CDC20 and CDH1 to prevent their activation of APC. APC was immunopurified from Xenopus interphase extracts; interphase APC only has a basal level of activity (Fig. 2A). Incubation of interphase APC with purified recombinant $\mathrm{CDC} 20$ and $\mathrm{CDH} 1$ activates its ubiquitin ligase activity; radioactively labeled cyclin B was quantitatively converted to high-molecular weight cyclin B-ubiquitin conjugates within 10 min (Fig. 2; Fang et al. 1998a). However, incubating recombinant MAD2B with $\mathrm{CDC} 20$ and CDH1 quantitatively blocks the activation of interphase APC by both CDC20 and CDH1. APC incubated with MAD2B plus CDC20 or plus $\mathrm{CDH} 1$ only has an activity comparable to interphase APC. On the other hand, when recombinant MAD2 was incubated with CDC20 and $\mathrm{CDH} 1, \mathrm{MAD} 2$ only blocks the activation of APC by CDC20, but not by CDH1, consistent with 


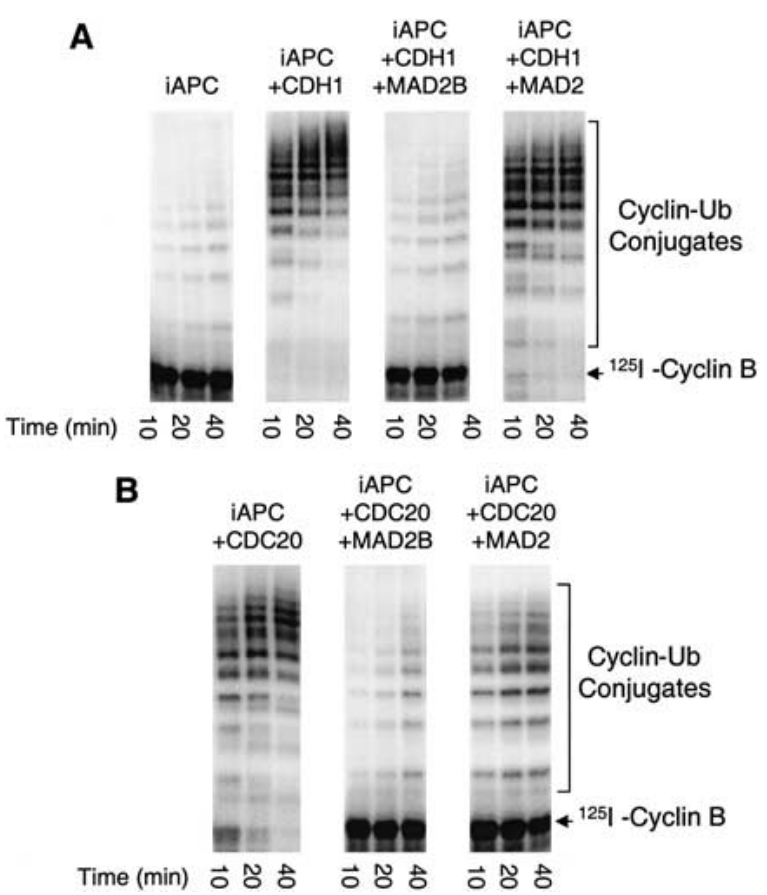

Figure 2. MAD2B directly inhibits activation of APC by CDC20 and CDH1. (A) Interphase APC (iAPC) was immunopurified from Xenopus extracts by anti-CDC27 antibody/protein A beads and then incubated with purified recombinant $\mathrm{CDH} 1$, $\mathrm{CDH} 1$ plus MAD2B or CDH1 plus MAD2. The ability of treated APC to ubiquitinate radioactive $\mathrm{N}$-terminal fragment of cyclin $\mathrm{B}$ was assayed. (B) Interphase APC was immunopurified by antiCDC27 antibody/protein A beads and then incubated with purified recombinant CDC20, CDC20 plus MAD2B or CDC20 plus MAD2. The ability of treated APC to ubiquitinate radioactive $\mathrm{N}$-terminal fragment of cyclin B was assayed.

previous reports that MAD2 is a specific inhibitor for CDC20 (Fang et al. 1998b; Hwang et al. 1998; Kim et al. 1998). Thus, MAD2B directly inhibits activation of APC by both CDC20 and CDH1.

Because MAD2B inhibits both $\mathrm{CDC} 20$ and $\mathrm{CDH} 1$ forms of APC, MAD2B may directly act on APC to prevent its activation by CDC20 and CDH1. This is unlikely, as its homolog MAD2 directly recognizes and inhibits CDC20, but not APC (Fang et al. 1998b; Hwang et al. 1998; Kim et al. 1998). To address this experimentally, we analyzed whether inhibition by MAD2B is directed to APC or to its activators CDC20 and CDH1. Immunopurified interphase APC was first incubated with recombinant MAD2B or MAD2. The recombinant proteins were then removed and the APC beads incubated with CDC20 (Fig. 3A, II and III). Prior incubation of interphase APC with MAD2B or MAD2 does not block its activation by CDC20. There are two possible explanations for this result. The inhibition by MAD2B may be directed either toward CDC20 or toward the CDC20APC complex, but not solely toward interphase APC. Alternatively, the inhibitory effect of MAD2B on APC may not be stable and the inhibition may be abolished during the second incubation with CDC20. To test the latter possibility, interphase APC was incubated with CDC20 and MAD2B or with CDC20 and MAD2, and the APC beads were then washed and incubated with buffer alone (Fig. 3A, IV and V). Both MAD2B and MAD2 still efficiently inhibit CDC20 after the second incubation with buffer alone, suggesting that the inhibitory effect of MAD2B and MAD2 is quite stable during the second incubation. These experiments suggest that inhibition by MAD2B is targeted toward CDC20 or toward the CDC20-APC complex. This is consistent with the fact that the target of MAD2-mediated inhibition is CDC20, not APC (Fang et al. 1998b; Hwang et al. 1998; Kim et al. 1998). Similarly, when interphase APC was first incubated with MAD2B and then with CDH1, MAD2B does not prevent activation of APC by CDH1 (Fig. 3B).

We then tested whether MAD2B can inhibit APC that had been activated by $\mathrm{CDC} 20$ and $\mathrm{CDH} 1$. Interphase APC was first activated by CDC20 or by CDH1, and MAD2B was then added and incubated with the mixture of APC and CDC20/CDH1. We found that once activated, MAD2B has no inhibitory effect on CDC20-APC and CDH1-APC (Fig. 3C; data not shown). Similarly, MAD2B does not inhibit mitotic CDC20-APC immunopurified from mitotic extracts (data not shown). Therefore, MAD2B blocks activation of APC by CDC20 and

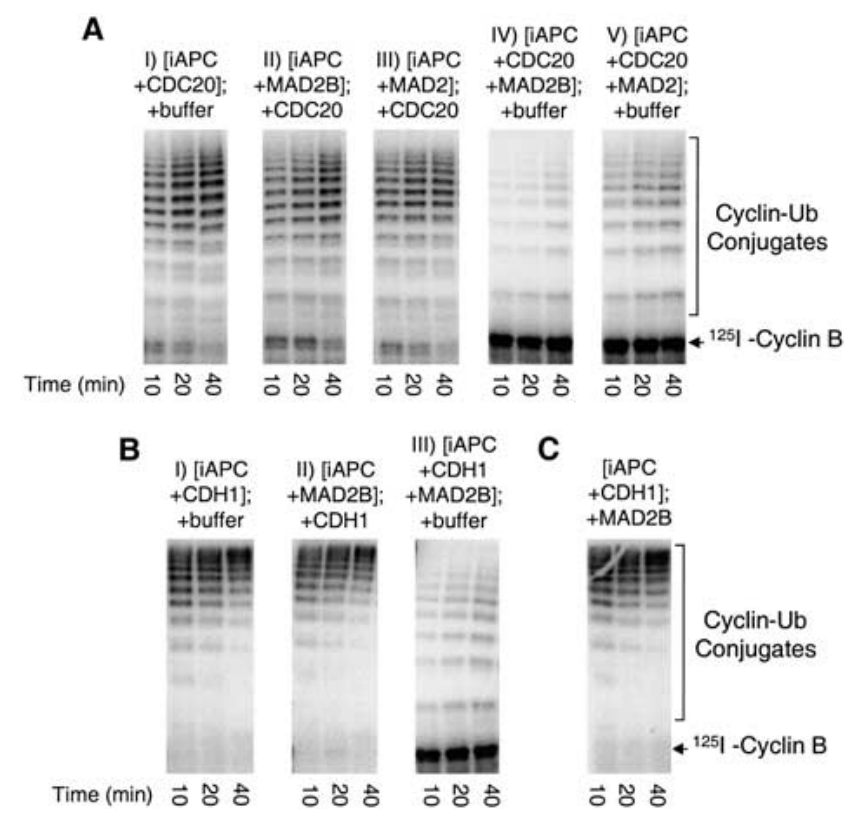

Figure 3. Inhibition by MAD2B is targeted to CDC20 and CDH1. (A) Interphase APC beads were incubated with purified recombinant CDC20 (I), with MAD2B (II), with MAD2 (III), with CDC20 plus MAD2B (IV), or with CDC20 plus MAD2 (V). Recombinant proteins were then removed and APC beads incubated with a buffer (I, IV, and V) or with recombinant CDC20 (II and III). The ability of treated APC to ubiquitinate radioactive $\mathrm{N}$-terminal fragment of cyclin $\mathrm{B}$ was assayed. $(B)$ Interphase APC beads were incubated with purified recombinant CDH1 (I), with MAD2B (II), or with CDH1 plus MAD2B (III). Recombinant proteins were then removed and APC beads incubated with a buffer (I and III) or with recombinant CDH1 (II). The ability of treated APC to ubiquitinate radioactive $\mathrm{N}$-terminal fragment of cyclin B was assayed. $(C)$ Interphase APC beads were first incubated with recombinant CDH1. MAD2B was then added and incubated with the mixture of CDH1 and APC. The ability of treated APC to ubiquitinate radioactive N-terminal fragment of cyclin B was assayed. 
CDH1, which have not been associated with APC, but does not inhibit the active CDC20-APC and CDH1APC complexes. This is consistent with the observation that MAD2 can only inhibit unbound, free CDC20, but not the activated CDC20-APC complex (data not shown). Thus, there is an apparent paradox. On one hand, MAD2B and MAD2 can inhibit APC in the mitotic extracts (see Fig. 1B). On the other hand, neither MAD2B nor MAD2 inhibits the active APC when immunopurified from mitotic extracts. We speculate that association of CDC20 and CDH1 with APC is dynamic in extracts and the turnover rate of the CDC20-APC and CDH1APC complexes in extracts may be regulated by cellular factors. This is consistent with the fact that CDC20 and $\mathrm{CDH} 1$ are substrate-specific activators of APC; they bind directly to substrates and target substrates to APC for ubiquitination (Schwab et al. 1997; Visintin et al. 1997; Fang et al. 1998a,b; Hwang et al. 1998; Kim et al. 1998).

\section{Inhibition of $A P C$ by $M A D 2 B$ is not specific to cyclin $B$}

The APC ubiquitinates multiple substrates in mitosis and $\mathrm{G}_{1}$. For example, both polo-like kinase (Plk1) and aurora-related kinase (ARK2) are degraded by the APC pathway as cells exit from mitosis (Charles et al. 1998; Fang et al. 1998a, 1999). Degradation of these mitotic kinases allows cells to reset the cell cycle state in $\mathrm{G}_{1}$. In addition, $\mathrm{CDC} 20$ is ubiquitinated by $\mathrm{CDH} 1-\mathrm{APC}$ at late mitosis and degradation of CDC20 leads to complete switch from CDC20-associated APC to CDH1-associated APC as cells exit from mitosis (Fang et al. 1998a). We tested whether inhibition of APC by MAD2B is specific to cyclin B. Plk1, ARK2, and CDC20 were translated in vitro and then incubated with interphase APC, with CDH1-APC and with APC, which had been incubated with recombinant MAD2B and CDH1 (Fig. 4). Interphase APC promotes a low level of ubiquitination of these substrates and CDH1-APC efficiently converts substrates into ubiquitin conjugates. In contrast, APC incubated with MAD2B and CDH1 only has an activity comparable to interphase APC for all the substrates analyzed (Fig. 4), indicating that the inhibitory effect by MAD2B is not restricted to cyclin B.

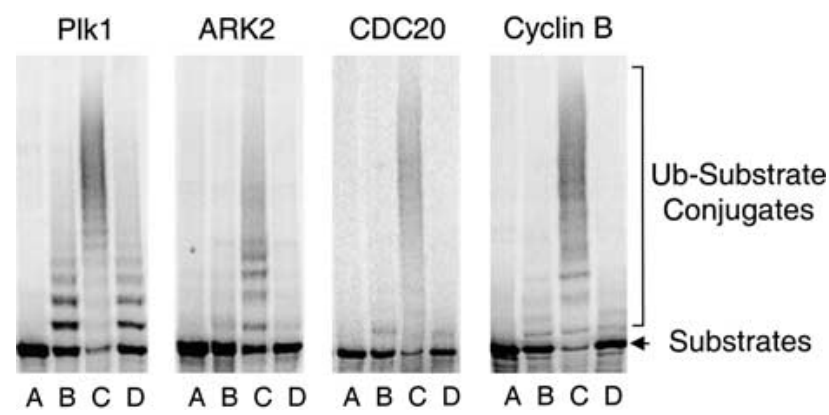

Figure 4. Inhibition of APC by MAD2B is not specific to cyclin $B$. Interphase APC (lanes $B$ ) was immunopurified by antiCDC27 antibody/protein A beads and then incubated with recombinant $\mathrm{CDH} 1$ (lanes $C$ ) or with $\mathrm{CDH} 1$ plus MAD2B (lanes $D)$. The ability of treated APC to ubiquitinate $\left[{ }^{35} \mathrm{~S}\right]$ Met-labeled Plk1, ARK2, CDC20, and full-length cyclin B was assayed.

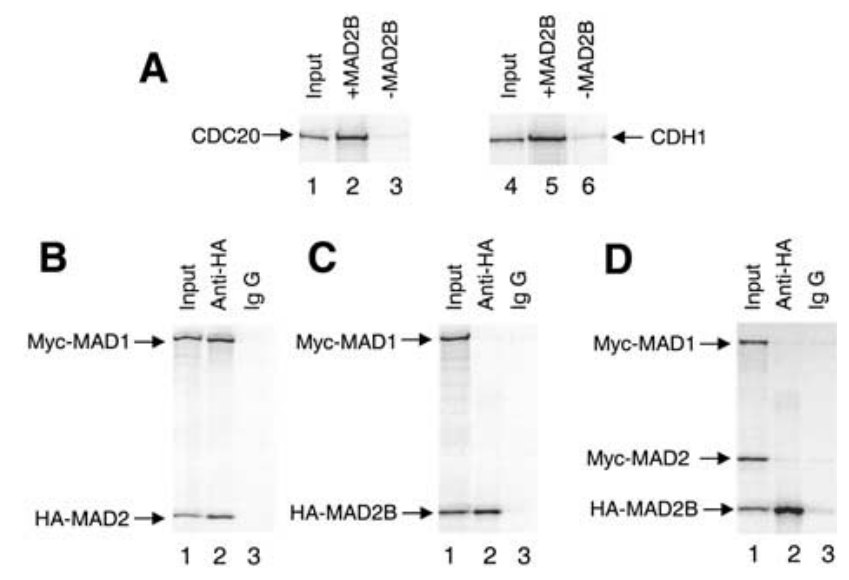

Figure 5. MAD2B interacts with $\mathrm{CDC} 20$ and $\mathrm{CDH} 1$, but not with MAD1. (A) His-MAD2B (lanes 2,4) or BSA (lanes 3,6) were incubated with ${ }^{35}$ S-labeled CDC20 (lanes 2,3) and CDH1 (lanes $5,6)$ and MAD2B complexes were purified by $\mathrm{Ni}^{+}$; beads and analyzed by SDS-PAGE. (Lanes 1,3) 1/10 of input CDC20 and CDH1. $(B-D)$ HA-MAD2 was incubated with Myc-MAD1 $(B)$. HA-MAD2B was incubated with Myc-MAD1 in the presence $(D)$ or absence $(C)$ of Myc-MAD2. HA-tagged proteins were then immunopurified by an anti-HA antibody (lane 2) or by control IgG (lane 3). The association of HA-tagged proteins with Myctagged proteins was analyzed by SDS-PAGE. (Lane 1) 1/10 of input proteins in the binding assay. In this experiment, six copies of the Myc tag were fused to the amino termini of MAD1 and MAD2 and two copies of the HA tag were fused to MAD2B. The large difference in the migration rate between Myc-MAD2 and HA-MAD2B was due to the difference in the copy number of the tags.

MAD2B interacts with both CDC20 and CDH1, but not with MAD1

MAD2 inhibits CDC20 through direct binding. Therefore, we tested whether MAD2B can directly associate with CDC20 and CDH1. ${ }^{35}$ S-labeled CDC20 and CDH1 were incubated with purified recombinant His-MAD2B or with BSA. MAD2B protein was then purified by $\mathrm{Ni}^{+}$ beads and associated proteins analyzed by SDS-PAGE (Fig. 5A). MAD2B directly binds to both CDC20 and $\mathrm{CDH} 1$, consistent with its ability to inhibit activation of APC by CDC20 and CDH1.

MAD2 interacts with the checkpoint protein MAD1 and formation of the MAD1-MAD2 complex is required for mitotic checkpoint control (Chen et al. 1999). Consistent with previous reports (Jin et al. 1998; Chen et al. 1999; Murakumo et al. 2000), we show here that immunoprecipitation of HA-MAD2 with an anti-HA antibody efficiently coprecipitated Myc-MAD1, indicating that MAD1 and MAD2 form a stable complex (Fig. 5B). However, when HA-MAD2B was incubated with MycMAD1, immunoprecipitation of HA-MAD2B by the anti-HA antibody did not bring down Myc-MAD1 (Fig. 5C). Similarly, immunoprecipitation with an anti-Myc antibody efficiently precipitated Myc-MAD1, but not HA-MAD2B (data not shown). It is possible that formation of the MAD1-MAD2 complex may be required for MAD1 and MAD2 to interact with MAD2B. To test this possibility, Myc-MAD1, Myc-MAD2, and HA-MAD2B were translated in vitro and then incubated together. Im- 
munoprecipitation with the anti-HA antibody efficiently precipitated HA-MAD2B, but neither Myc-MAD2 nor Myc-MAD1, indicating that MAD2B does not interact with the MAD1-MAD2 complex (Fig. 5D). Thus, MAD2B does not associate with the checkpoint proteins MAD1 and MAD2. We speculate that MAD2B is likely involved in a cellular process other than the spindle assembly checkpoint control.

\section{Discussion}

The APC controls both sister chromatid separation and exit from mitosis by ubiquitinating several key cell cycle regulators in mitosis and in $\mathrm{G}_{1}$ (Fang et al. 1999). The activity of APC is precisely regulated by a network of regulatory factors that include two activators, CDC20 and CDH1, as well as an inhibitor, MAD2. We show here that a homolog of MAD2, MAD2B, is also an inhibitor of APC. MAD2B directly binds to CDH1 and CDC20 and prevents activation of APC by CDH1, and to a lesser extent, by CDC20, both in extracts as well as in a reconstituted system. Like MAD2, inhibition of APC by MAD2B requires stoichiometric amounts of MAD2B relative to $\mathrm{CDC} 20$ and $\mathrm{CDH} 1$ (Fig. 1; data not shown). This inhibition is quantitative, as MAD2B reduces the APC activity to a level comparable to that of interphase APC. The inhibition is at least partially targeted to CDH1 and CDC20, as preincubation of MAD2B with interphase APC does not block subsequent activation by CDH1 and CDC20. Furthermore, MAD2B has a broad inhibitory effect on the APC activity and inhibits ubiquitination of all four different substrates tested.

The specificity of MAD2B-mediated inhibition is different from that of MAD2, a component of the spindle assembly checkpoint pathway (Fang et al. 1998b; Hwang et al. 1998; Kim et al. 1998). Whereas MAD2 only inhibits CDC20-APC, MAD2B inhibits CDH1, and to a lesser extent, CDC20 forms of APC. This different spectrum of inhibitory specificity between MAD2B and MAD2 is likely to reflect different physiological function of the two proteins. The spindle assembly checkpoint monitors the attachment of the mitotic spindle to kinetochores and prevents premature separation of sister chromatids in the presence of unattached kinetochores (Amon 1999). Because activation of APC by CDC20 at the metaphase to anaphase transition leads to degradation of securin and separation of sister chromatids, CDC20-APC is the target for mitotic checkpoint control (Fang et al. 1998b; Hwang et al. 1998; Kim et al. 1998). The checkpoint protein MAD2 inhibits CDC20 and APC by forming an inactive binary MAD2-CDC20 as well as a ternary MAD2-CDC20-APC complex, and thereby arrests cells at metaphase. CDH1 is phosphorylated by the CDC2 kinase at early mitosis and phosphorylated $\mathrm{CDH1}$ is in an inactive state that fails to bind to and activate APC (Zachariae et al. 1998). Thus, the MAD2 pathway only targets CDC20 for inactivation. MAD2 is localized only to those kinetochores that have not been attached to mitotic spindle, indicating a critical function of unattached kinetochores in checkpoint control (Chen et al. 1996; Li and Benezra 1996). This kinetochore localization is mediated through the checkpoint protein MAD1 and the inhibitory effect of MAD2 requires its interaction with MAD1 (Chen et al. 1998, 1999). In contrast, MAD2B is a nuclear protein based on studies with the GFP-MAD2B fusion protein (data not shown). No spe- cific kinetochore association was detected in prophase and premetaphase cells as well as in nocodazole-treated metaphase cells. Furthermore, unlike MAD2, MAD2B does not interact with MAD1. These data coupled with the fact that MAD2B can inhibit both CDC20 and CDH1 forms of APC suggest that MAD2B is likely involved in a cellular process other than the spindle assembly checkpoint control.

What is the physiological function of MAD2B? MAD2B is a highly conserved protein in vertebrates and is expected to function in a highly conserved physiological process (Cahill et al. 1999). Homologs of MAD2B have been found in Xenopus, mouse, and human through BLAST search of GenBank database (data not shown); the Xenopus and human MAD2B proteins share 95\% identity at the amino acid level, a degree of homology much higher than that between Xenopus and human MAD2 (80\% identity). The Xenopus MAD2B has also been shown to inhibit APC (Pfleger et al. 2001). Interestingly, Xenopus MAD2B only inhibits CDH1-APC, but not CDC20-APC. The functional importance of this differential inhibitory specificity between human and Xenopus proteins remains a topic for investigation.

MAD2B may control the timing of cytokinesis. This is a cell cycle stage where sister chromatids have already been separated by CDC20-APC-dependent proteolysis, but the CDH1 has yet to activate APC to complete cell division and to exit from mitosis (Fang et al. 1999; Zachariae and Nasmyth 1999). Thus, both CDC20 and CDH1 are present at this stage of the cell cycle. Cells may have a surveillance mechanism that monitors the completion of sister chromatid separation, the orientation of mitotic spindle, or the structure of the cleavage furor before activating the CDH1-APC complex for cytokinesis and for exit from mitosis. It is conceivable that MAD2B may play a role in regulating both CDC20 and $\mathrm{CDH} 1$ activity in such a surveillance mechanism. In fact, such a checkpoint mechanism has been reported in yeast (Muhua et al. 1998; Wang et al. 2000). This late mitotic checkpoint delays exit from mitosis when the mitotic spindle is orientated abnormally. Because cytokinesis is regulated very differently between yeast and vertebrates, we expect that the molecular details for the late mitotic checkpoint pathway in vertebrates may be different from yeast.

Alternatively, MAD2B may function in a physiological process other than cell cycle regulation. The activity of APC is not restricted to proliferating cells and APC is also active in terminally differentiated cells, such as neurons (Gieffers et al. 1999). In fact, APC immunopurified from brain is as active as APC from mitotic and $G_{1}$ cells. The activity of APC is maintained by CDH1 in terminally differentiated cells. It is conceivable that MAD2B may play a role in the control of the APC activity in these differentiated tissues. Although our biochemical analysis of MAD2B uncovered an important activity of this protein, a firm establishment of the physiological role of MAD2B awaits detailed characterization of the expression profile and the function of this protein in vivo.

\section{Materials and methods}

Molecular cloning of MAD2B gene and expression and purification of recombinant proteins

Human $M A D 2 B$ gene was cloned by PCR amplification from human fetal thymus cDNA, subcloned into pET28a (Novagen), and expressed as in- 
clusion bodies in BL21(DE3)pLys Codon Plus (Strategene). Similarly, $M A D 2 B$ gene was also subcloned into pFastBac (GIBCO BRL) and expressed as inclusion bodies in $S f 9$ cells. The recombinant protein was purified by Ni-NTA beads under denaturing conditions and refolded with a linear gradient of decreasing urea concentration. The soluble recombinant protein was further purified as a single symmetric peak on a HiTrap $\mathrm{Q}$ column. The purified protein was dialyzed against a buffer containing $10 \mathrm{mM}$ Tris-Cl (pH 7.7), $100 \mathrm{mM} \mathrm{KCl}$, and $1 \mathrm{mM}$ DTT and used in experiments described in this paper. We also expressed and purified MAD2B fused to glutathione-S-transferase (GST) in the pGEX-4T-1 vector. A small portion of the recombinant GST-MAD2B protein was soluble, but the GST fusion interferes with the folding of MAD2B, as MAD2B remains associated with GST after cleavage at the fusion junction by a protease (data not shown). Expression and purification of recombinant MAD2, CDC20, and $\mathrm{CDH} 1$ were described previously (Fang et al. 1998b, 1999).

Cyclin degradation and ubiquitination assays

To assay cyclin degradation in crude mitotic extracts, $2 \mu \mathrm{L}$ of MAD2B, MAD2, or a buffer was added to $8 \mu \mathrm{L}$ of Xenopus mitotic extracts and incubated at room temperature for $1 \mathrm{~h}$. To assay the effect of MAD2B on CDH1-APC in interphase extracts, $1 \mu \mathrm{L}$ of recombinant CDH1 $11 \mathrm{mg} /$ $\mathrm{mL}$ ) and $2 \mu \mathrm{L}$ of MAD2B or a buffer were incubated with $7 \mu \mathrm{L}$ of Xenopus interphase extracts for $1 \mathrm{~h}$. The final concentrations for MAD2B and MAD2 proteins in extracts were $0.5,0.25$, or $0.125 \mathrm{mg} / \mathrm{mL}$ and the concentration for $\mathrm{CDH} 1$ was at $0.1 \mathrm{mg} / \mathrm{mL}$. Cyclin degradation assay was performed as described previously (Fang et al. 1998b).

To assay effects of MAD2B and MAD2 on activation of APC in reconstituted ubiquitination reactions in vitro, APC was first immunopurified from Xenopus extracts by anti-CDC27 antibody/protein A beads and then incubated with CDC20, CDH1, MAD2, MAD2B in orders specified in the figure legends. Purified APC were then assayed for the ubiquitin ligase activity (Fang et al. 1998b).

\section{Acknowledgments}

We thank Dr. James Ferrell for helpful comments on the manuscript. G.F. is a Searle Scholar and a recipient of the Beckman Young Investigator Award. This work was also supported by a Career Award from the Burroughs Wellcome Fund and by a research grant from Concern Foundation to G.F.

The publication costs of this article were defrayed in part by payment of page charges. This article must therefore be hereby marked "advertisement" in accordance with 18 USC section 1734 solely to indicate this fact.

\section{References}

Amon, A. 1999. The spindle checkpoint. Curr. Opin. Genet. Dev. 9: 69-75.

Amon, A., Irniger, S., and Nasmyth, K. 1994. Closing the cell cycle circle in yeast: G2 cyclin proteolysis initiated at mitosis persists until the activation of G1 cyclins in the next cycle. Cell 77: 1037-1050.

Cahill, D.P., da Costa, L.T., Carson-Walter, E.B., Kinzler, K.W., Vogelstein, B., and Lengauer, C. 1999. Characterization of MAD2B and other mitotic spindle checkpoint genes. Genomics 58: 181-187.

Charles, J.F., Jaspersen, S.L., Tinker-Kulberg, R.L., Hwang, L., Szidon, A., and Morgan, D.O. 1998. The Polo-related kinase Cdc5 activates and is destroyed by the mitotic cyclin destruction machinery in S. cerevisiae. Curr. Biol. 8: 497-507.

Chen, R.H., Waters, J.C., Salmon, E.D., and Murray, A.W. 1996. Association of spindle assembly checkpoint component XMAD2 with unattached kinetochores. Science 274: 242-246.

Chen, R.H., Shevchenko, A., Mann, M., and Murray, A.W. 1998. Spindle checkpoint protein Xmad1 recruits Xmad2 to unattached kinetochores. J. Cell Biol. 143: 283-295.

Chen, R.H., Brady, D.M., Smith, D., Murray, A.W., and Hardwick, K.G. 1999. The spindle checkpoint of budding yeast depends on a tight complex between the mad1 and mad2 proteins. Mol. Biol. Cell 10: $2607-2618$.

Fang, G., Yu, H., and Kirschner, M.W. 1998a. Direct binding of CDC20 protein family members activates the anaphase-promoting complex in mitosis and G1. Mol. Cell 2: 163-171.

- 1998b. The checkpoint protein MAD2 and the mitotic regulator CDC20 form a ternary complex with the anaphase-promoting complex to control anaphase initiation. Genes \& Dev. 12: 1871-1883.

- 1999. Mitotic proteolysis. Philo. Trans. R. Soc.: Biol. Sci. 354: 1583-1590.

Gieffers, C., Peters, B.H., Kramer, E.R., Dotti, C.G., and Peters, J.M. 1999 Expression of the $\mathrm{CDH} 1$-associated form of the anaphase-promoting complex in postmitotic neurons. Proc. Natl. Acad. Sci. 96: 1131711322.

Glotzer, M., Murray, A.W., and Kirschner, M.W. 1991. Cyclin is degraded by the ubiquitin pathway. Nature 349: 132-138.

Hwang, L.H., Lau, L.F., Smith, D.L., Mistrot, C.A., Hardwick, K.G., Hwang, E.S., Amon, A., and Murray, A.W. 1998. Budding yeast Cdc20: A target of the spindle checkpoint. Science 279: 1041-1044.

Jin, D.Y., Spencer, F., and Jeang, K.T. 1998. Human T cell leukemia virus type 1 oncoprotein Tax targets the human mitotic checkpoint protein MAD1. Cell 93: 81-91.

Kim, S.H., Lin, D.P., Matsumoto, S., Kitazono, A., and Matsumoto, T. 1998. Fission yeast Slp1: An effector of the Mad2-dependent spindle checkpoint. Science 279: 1045-1047.

King, R.W., Peters, J.M., Tugendreich, S., Rolfe, M., Hieter, P., and Kirschner, M.W. 1995. A 20S complex containing CDC27 and CDC16 catalyzes the mitosis-specific conjugation of ubiquitin to cyclin B. Cell 81: $279-288$

Li, Y. and Benezra, R. 1996. Identification of a human mitotic checkpoint gene: hsMAD2. Science 274: 246-248.

Lorca, T., Castro, A., Martinez, A.M., Vigneron, S., Morin, N., Sigrist, S., Lehner, C., Doree, M., and Labbe, J.C. 1998. Fizzy is required for activation of the APC/cyclosome in Xenopus egg extracts. EMBO J. 17: 3565-3575.

Muhua, L., Adames, N.R., Murphy, M.D., Shields, C.R., and Cooper, J.A 1998. A cytokinesis checkpoint requiring the yeast homologue of an APC-binding protein. Nature 393: 487-491.

Murakumo, Y., Roth, T., Ishii, H., Rasio, D., Numata, S., Croce, C.M., and Fishel, R. 2000. A human REV7 homolog that interacts with the polymerase zeta catalytic subunit hREV3 and the spindle assembly checkpoint protein hMAD2. J. Biol. Chem. 275: 4391-4397.

Pfleger, C.M. and Kirschner, M.W. 2000. The KEN box: An APC recog nition signal distinct from the D box targeted by cdh1. Genes \& Dev 14: $655-665$.

Pfleger, C.M., Salic, A., Lee, E., and Kirschner, M.W. 2001. Inhibition of Cdh1-APC by the MAD2-related protein MAD2L2: A novel mechanism for regulating Cdh1. Genes \& Dev. 15: (this issue).

Schwab, M., Lutum, A.S., and Seufert, W. 1997. Yeast Hct1 is a regulator of Clb2 cyclin proteolysis. Cell 90: 683-693.

Sudakin, V., Ganoth, D., Dahan, A., Heller, H., Hershko, J., Luca, F.C., Ruderman, J.V., and Hershko, A. 1995. The cyclosome, a large complex containing cyclin-selective ubiquitin ligase activity, targets cyclins for destruction at the end of mitosis. Mol. Biol. Cell. 6: 185-197.

Visintin, R., Prinz, S., and Amon, A. 1997. CDC20 and CDH1: A family of substrate-specific activators of APC- dependent proteolysis. Science 278: 460-463.

Visintin, R., Craig, K., Hwang, E.S., Prinz, S., Tyers, M., and Amon, A. 1998. The phosphatase Cdc14 triggers mitotic exit by reversal of Cdkdependent phosphorylation. Mol. Cell 2: 709-718.

Wang, Y., Hu, F., and Elledge, S.J. 2000. The Bfa1/Bub2 GAP complex comprises a universal checkpoint required to prevent mitotic exit Curr. Biol. 10: 1379-1382.

Zachariae, W. and Nasmyth, K. 1999. Whose end is destruction: cell division and the anaphase-promoting complex. Genes \& Dev. 13: 2039-2058.

Zachariae, W., Schwab, M., Nasmyth, K., and Seufert, W. 1998. Control of cyclin ubiquitination by CDK-regulated binding of Hctl to the anaphase promoting complex. Science 282: 1721-1724. 


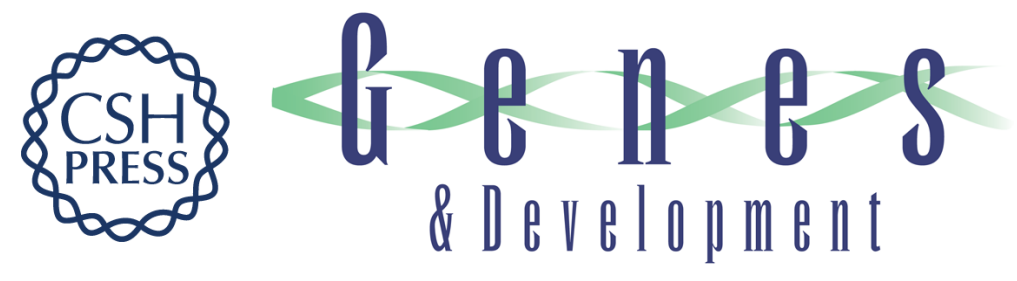

\section{MAD2B is an inhibitor of the anaphase-promoting complex}

James Chen and Guowei Fang

Genes Dev. 2001, 15:

Access the most recent version at doi:10.1101/gad.898701

References This article cites 28 articles, 15 of which can be accessed free at: http://genesdev.cshlp.org/content/15/14/1765.full.html\#ref-list-1

License

Email Alerting Receive free email alerts when new articles cite this article - sign up in the box at the top Service right corner of the article or click here.

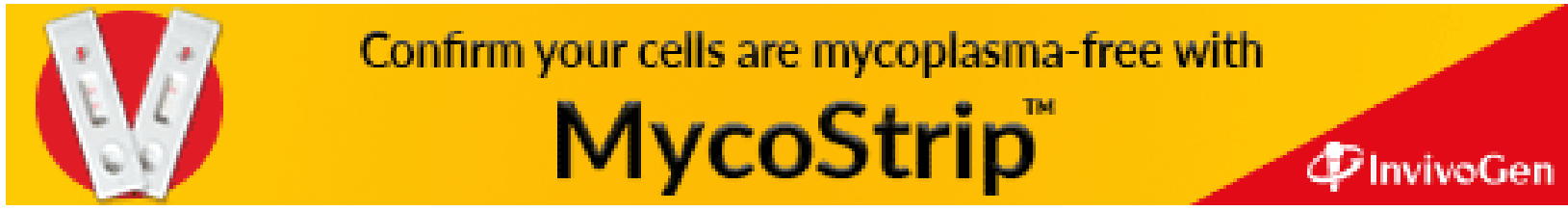

